\title{
Vivables, vivantes et vivrières : de nouveaux espoirs pour la ville?
}

Lively, livable, life supplying: new hopes for cities?

\section{Julien Blanc, Bernadette Lizet et Françoise Dubost}

\section{(2) OpenEdition}

1 Journals

Édition électronique

URL : http://journals.openedition.org/ethnoecologie/2629

DOI : $10.4000 /$ ethnoecologie.2629

ISSN : 2267-2419

Éditeur

Laboratoire Eco-anthropologie et Ethnobiologie

Référence électronique

Julien Blanc, Bernadette Lizet et Françoise Dubost, «Vivables, vivantes et vivrières : de nouveaux espoirs pour la ville? », Revue d'ethnoécologie [En ligne], 8 | 2015, mis en ligne le 04 juillet 2016, consulté le 30 avril 2019. URL : http://journals.openedition.org/ethnoecologie/2629 ; DOI : 10.4000/ ethnoecologie.2629

Ce document a été généré automatiquement le 30 avril 2019.

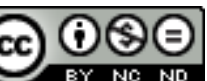

Revue d'ethnoécologie est mis à disposition selon les termes de la licence Creative Commons Attribution - Pas d'Utilisation Commerciale - Pas de Modification 4.0 International. 


\section{Vivables, vivantes et vivrières : de nouveaux espoirs pour la ville?}

Lively, livable, life supplying: new hopes for cities?

Julien Blanc, Bernadette Lizet et Françoise Dubost

1 Nous abordons le sujet sur la base des recherches sur les natures urbaines lancées au Muséum dans les années 1990, concrétisées par un colloque (1996), deux journées d'études et deux opérations de recherche au sein de l'UMR Éco-Anthropologie et Ethnobiologie, le tout chevillé à des enseignements ${ }^{1}$. La problématique initiale était celle des « villes naturalisées ", qui a été commuée en «villes vivrières ». L'idée majeure qui se dégage de cette réflexion collective est l'enchaînement des représentations de la ville idéale, des transformations à entreprendre pour faire face aux grands déséquilibres écologiques. Or, l'agriculture urbaine semble aujourd'hui s'être imposée comme une référence majeure pour la construction des villes durables remplaçant, en grande partie tout du moins, - ou l'englobant peut-être ? - la référence d'hier : la biodiversité. Voilà une dizaine d'années, nous nous posions la question de l'enchaînement des idéologies et de leurs hybridations, « De l'hygiénisme au développement durable » (Dubost \& Lizet 2003). Aujourd'hui nous nous interrogeons sur la manière dont la problématique de la nature en ville et du vivant s'arrime à celle de l'agriculture urbaine, dans les politiques comme dans les pratiques des habitants et des mouvements associatifs. Il était important d'identifier les modèles d'agriculture qui se déploient d'un bout à l'autre de la planète, les enjeux auxquels ils sont censés répondre à chaque fois et en chaque lieu, autant que la manière dont ils s'incarnent et circulent dans la vie des villes. Dans chaque situation, il faut comprendre comment la nature est convoquée (et de quelle nature il s'agit), identifier les représentations et les traitements de la nature inclus dans les différents modèles d'agriculture urbaine.

Ce dossier a été plus particulièrement constitué autour de l'idée de villes "vivrières ". Nous avons invité les auteurs à considérer le double sens du mot "vivre» (les vivresaliments et le vivre-vécu) : la dimension nourricière de cette agriculture pour les villes étudiées (en Espagne, au Niger, au Bénin, en Argentine, en Russie, en France, en Suisse), 
mais aussi sa dimension symbolique (l'aliment et le vivant, humain et non humain) et plus globalement son rôle structurel dans l'organisation de la vie urbaine. Si la plupart des textes ne traitent pas directement des modes d'articulation des problématiques de la nature en ville et de l'agriculture urbaine, ils fournissent de nombreux points d'accroche pour mener la réflexion. Et ils nous en apprennent beaucoup sur les manières d'habiter la ville et de la «faire».

\section{Sous la bannière de l'agriculture urbaine}

S'y trouvent rassemblées des innovations et des pratiques plus anciennes bénéficiant d'un regain d'intérêt. Elles ont en commun d'être propulsées au-devant de la scène dans le contexte des crises environnementales, économiques et urbaines, parce qu'elles s'affirment comme autant de réponses potentielles à l'enjeu majeur du « développement durable » et de lutte contre la pauvreté. Les protagonistes et promoteurs de ces activités sont de simples citoyens, des acteurs de la société civile organisée (associations, ONG), des représentants des pouvoirs publics ou des entrepreneurs. Ils invoquent pour justifier leur action une contribution à la durabilité environnementale des villes, une réponse (partielle) aux problèmes de sécurité alimentaire des populations urbaines les plus défavorisées, le moyen de mieux se nourrir face à la piètre qualité et à la dangerosité des aliments issus de l'agro-industrie. Dans des contextes de décrépitude de l'action publique (libéralisation, faiblesse des pouvoirs publics) ou au contraire en résistance au fait institutionnel, cette agriculture-là a même parfois clairement l'ambition d'une réappropriation de la fabrique urbaine, de la fabrique alimentaire et de la fabrique sociale. Car si l'agriculture urbaine est une pratique agricole et jardinière ${ }^{2}$, c'est aussi pour beaucoup une pratique politique.

Ce qu'on trouve aujourd'hui rassemblé derrière «l'agriculture urbaine» renvoie de manière croissante à une activité organisée, planifiée par le haut ou orchestrée par le bas, et bien souvent les deux à la fois. Il serait plus précis de dire que cette activité s'organise de plus en plus dans la tension entre un foisonnement d'expériences et de créativité urbaine (Mougeot 2006) et leur institutionnalisation (Dubbeling \& Van Veenhuizen 2014). Qu'on l'appréhende globalement ou localement, l'agriculture urbaine implique une diversité grandissante d'acteurs, d'espaces, de ressources financières et de réseaux d'échange de savoirs et de pratiques. Les démarches sont complexes, et les analyses doivent prendre en compte cette complexité, dans une vision systémique ou " rhizomique $»^{3}$ (selon la terminologie et l'analogie botanique lancées en 1980 par Deleuze et Guattari). La référence insistante chez les jardiniers urbains du Nord à la permaculture, qui conjugue philosophie, esthétique, écologie et technique et mobilise systématiquement les notions de système et de rhizome, nous apparaît particulièrement significative à cet égard ${ }^{4}$.

Les contributions de ce dossier ont été choisies pour faire varier à la fois l'échelle (d'espace et de temps) et l'angle d'appréhension du phénomène. Développant une réflexion à partir du "micro ", l'approche ethnographique (Jacqueline Milliet, Antoine Quenardel, Julie Scapino), révèle la particularité de chaque cas de figure, en prenant en compte ce qui se joue dans l'arrière-scène, entre le singulier et l'universel, dans l'entrecroisement des plans du social, du politique et de l'économique, de l'idéel, du matériel et du symbolique. D'autres auteurs ont d'emblée ancré leurs analyses à large échelle, avec une visée mondiale et typologiste (Silvia Pérez-Vitoria), ou dans l'espace 
national (Louiza Boukharaeva et Marcel Marloie). D’autres encore (Dominique JuhéBeaulaton, Anne Luxereau, Marta Soler, Raúl Puente Asuero, Laurence Baudelet) jouent à la fois sur les tableaux du national et du local. Cette hétérogénéité a permis d'éclairer les différentes facettes du phénomène et de souligner la grande diversité des enjeux, des formes d'engagements et des problématiques qui s'y rattachent. La prise en compte du passé permet de mettre en évidence ce qui est vraiment spécifique à notre époque : une représentation de l'agriculture urbaine, par exemple, qui s'affirme comme de la « nature vivrière ", une agriculture résolument jardinière et écologique (Jacqueline Milliet, Julie Scapino, Marta Soler, Raúl Puente Asuero). Elle permet également de réfléchir sur des processus au long cours, comme l'évincement de l'agriculture du tissu urbain et périurbain et son retour aujourd'hui (Antoine Quenardel, Raúl Puente Asuero) ou la résistance du mode de vie individualiste lié aux datchas, qui suscitait pourtant la méfiance des soviétiques (Louiza Boukharaeva et Marcel Marloie). Quant à la persistance de la production agricole domestique dans les villes anciennes puissantes d'Abomey et de Ouidah, elle s'explique par une politique coloniale visant à les mettre à l'écart de certains circuits commerciaux (Dominique Juhé-Beaulaton). Plusieurs auteurs étudient des phénomènes actuels, comme la création de toutes pièces d'une ceinture maraîchère et fruitière autour des villes moyennes et des bourgs en Afrique de l'Ouest (Anne Luxereau), la diffusion du modèle constitué par le jardin écologique et social de Miraflores à travers la capitale andalouse et l'Espagne urbaine (Raúl Puente Asuero), mais aussi dans le monde latino-américain, par exemple à Rosario en Argentine (Marta Soler).

\section{Du nouveau sur la frontière entre villes et campagnes}

Les auteurs s'interrogent sur les limites toujours plus floues et sans cesse négociées entre les villes et les campagnes, le rural et l'urbain. La thématique est rebattue, mais le vigoureux mouvement de l'agriculture urbaine la renouvelle. Raúl Puente Asuero y voit un retournement (une diversification?) du processus néo-rural qui marque l'Europe médiane et méridionale depuis les années 1980 : pour vivre plus sainement, de plus en plus de familles choisissent de «transférer la campagne » dans leurs lieux urbains, plutôt que d'aller habiter dans un village. Elles «créent des bulles rurales au sein d'une mer urbaine » : c'est la campagne qui entrerait (ferait un retour) en ville. Considérant la précarisation de l'agriculture paysanne dans le monde face aux lobbys industriels et à l'expansion urbaine, Silvia Pérez-Vitoria met en garde contre la séduction exercée par l'agriculture urbaine, qui polarise l'attention et jetterait un masque sur la situation des petits paysans, qui fournissent toujours une très grande majorité de l'alimentation des hommes. Pour Anne Luxereau, la vitalité sans précédent des cultures maraîchères et fruitières, dans les villes africaines et leurs périphéries plus ou moins proches, dans les campagnes également, trouve son explication dans la fragilité de l'agriculture pluviale depuis les années 1970-1980 et le fort exode rural; elle souligne la capacité des paysans nigériens à « se saisir des opportunités créées par la croissance urbaine et la création de liaisons routières et fluviales ». Ces jardins irrigués, partout où c'est possible dans toute l'Afrique de l'Ouest, effacent d'une certaine manière la frontière entre l'urbain et le rural, recomposent les complémentarités entre ces espaces tout en créant des disparités nouvelles entre territoires.

7 Tous les autres articles sont centrés sur des espaces urbains, où les «bulles rurales » prennent des formes très diverses et s'installent dans des lieux aux statuts contrastés. 
Tout un éventail de potagers publics, écologiques et sociaux « fait » Séville aujourd'hui, de l'immense dépotoir en périphérie au centre historique ${ }^{5}$, en passant par l'enceinte d'un collège et d'une université, d'un hôpital psychiatrique abandonné. Dans les villes bourgeoises de l'arc lémanique, en Suisse, on élève des vers recycleurs de déchets au salon, dans un meuble qui leur est dédié, le lombricomposteur. Petits univers familiaux et privés, à la fois productifs et paysagers, les datchas sont au contraire éloignées du lieu de résidence, tout comme les jardins de Saulx-les-Chartreux, dans l'Essonne, où se maintient pareillement une pratique familiale, ancienne et populaire. L'activité n'y est pas marchande et elle s'oppose sur de multiples points au maraîchage professionnel environnant, reliquat de l'ancienne ceinture maraîchère parisienne qui a en général très mal résisté à la périurbanisation. À Paris un groupe d'artistes et « d'activistes du végétal » s'est approprié un bout de la friche ferroviaire de la petite ceinture, et met en scène une «nature nourricière » dans un espace sec et pollué, symboliquement antinomique de la vie. C'est d'ailleurs cette contradiction apparente entre le désir des urbains de renouer avec la terre, le végétal et la production dans un environnement hostile à la vie, car notamment pollué, qu'interroge Laurence Baudelet. À Niamey (Niger) et à Cotonou (Bénin), un nouveau maraîchage colonise une multitude d'interstices et de franges urbaines pour répondre à une consommation citadine en hausse constante: dans un contexte de compétition foncière extrême, les délaissés y sont mis en cultures potagères et fruitières par les habitants.

Sur ces ponts tendus entre villes et campagnes, circulent des savoirs. En Afrique de l'Ouest le maraîchage apparaît comme intermédiaire sur tous les plans. Sa vitalité et sa visibilité croissante dans le paysage rural et urbain vont de pair avec une professionnalisation de l'activité en ville, qui puise dans le vieux fonds des compétences et des semences paysannes, mais s'affirme surtout dans une capacité de chacun à se prendre en charge, à produire des légumes et des fruits partout où c'est possible et d'une manière la plus intensive possible, en combinant les ressources locales et exogènes (espèces et variétés, pratiques culturales). Le "jardin de ballast » réalisé sur la friche ferroviaire de la petite ceinture à Paris est un pur produit urbain en réseau planétaire, avant tout contestataire et démonstratif d'une vision alternative du monde. Dans ce lieu stérile et en principe interdit d'accès, les activistes produisent un système écologique et idéologique très structuré, dont l'aliment constitue clairement un sous-produit et un symbole. La visée des parcs-jardins de Rosario est, quant à elle, sociale (comme à Séville) : il faut aider les plus pauvres à s'organiser collectivement pour tirer de la terre urbaine de quoi mieux manger et mieux vivre (emploi et revenu, identité personnelle et collective), en socialisant l'activité (désenclaver le jardinage social en l'insérant dans des parcs publics). Tous ces repères pour l'action sont présents dans le modèle sévillan de Miraflores, mais le programme de Rosario possède une singularité : la culture de légumes est un levier pour récupérer et valoriser les savoirs paysans des immigrés pauvres, chassés des campagnes par le productivisme agricole.

\section{Crise durable et mondes possibles}

9 C'est devenu une banalité de dire que la crise est multiforme, et que l'enjeu de la "durabilité » de la planète habitée, par les humains et les autres espèces, consiste à tendre vers une maîtrise harmonieuse des facteurs économiques, sociaux et environnementaux. Cultiver la ville, lieu de vie d'une majorité croissante d'êtres humains 
sur la terre, c'est donc s'affronter aux grands problèmes. Au raisonnement sur les trois "piliers" du développement durable, fait écho le constat d'un caractère «multifonctionnel» de l'agriculture urbaine, qui serait donc, par essence, durable. Qu'apporte notre dossier sur cette problématique? Quelle place les chercheurs lui ont-ils accordée dans leurs textes et quels sont les acteurs, sur leurs terrains respectifs, qui défendent cette démarche d'analyse et d'action en triptyque? Quelle position les chercheurs adoptent-ils eux-mêmes à cet égard ?

Dans son tour d'horizon planétaire, Silvia Pérez-Vitoria souligne les enjeux de souveraineté alimentaire et leur rôle dans le développement de l'agriculture urbaine, tout particulièrement dans les bidonvilles: plus d'un milliard d'êtres humains y vivent aujourd'hui et ils seront selon toutes prévisions deux milliards en 2030 (UN-Habitat 2003). Il est classique d'établir une ligne de démarcation entre le Sud et le Nord selon la place accordée à la production de nourriture : majeure au Sud, pour les plus démunis, et nettement subordonnée au Nord à d'autres finalités (faire du lien social et donner l'exemple, embellir, restaurer par la nature et rétablir les liaisons écologiques...). Silvia Pérez-Vitoria ne remet pas ce grand clivage en question, mais elle affirme que le chômage et la pauvreté croissante donnent « une certaine centralité à la question alimentaire dans les villes les plus riches ", ce que Françoise Dubost (2011) a bien repéré, en France, dans les jardins potagers collectifs. Les éleveurs de vers de Lausanne et de Neuchâtel étudiés ici par l'ethnologue Jacqueline Milliet (très distanciée de son objet d'analyse) sont par contre totalement à l'abri du besoin de nourriture; ils appartiennent à une couche sociale nettement favorisée, et par ailleurs très encadrée par les institutions territoriales. Les cantons ont eu la charge d'appliquer une loi fédérale sur la protection de l'environnement précoce en Europe: dès 1983, elle programmait une organisation du ramassage et du compostage domestique des déchets. Le plaisir manifeste des habitants à fabriquer de la « terre urbaine » dans leurs lombricomposteurs grouillants combine deux registres : agir en bons citoyens pour la planète par la transformation d'une nuisance en ressource et s'insérer dans une chaîne sociale de fabrication et d'utilisation de l'«or noir ». Pas trace de système de discours et de représentation de la durabilité chez les jardiniers du sud de l'Essonne, qui profitent en famille et dans le voisinage, de génération en génération, de leurs petits édens conquis sur les bois (et plus ou moins reconquis par les bois). Des légumes, des fruits, mais aussi et surtout sans doute, du plaisir d'y être ensemble pour cultiver, manger, ne rien faire, regarder... C'est le chercheur, paysagiste de formation, qui considère l'échiquier écologique et social constitué par l'ensemble de ces petites parcelles, y décèle de la valeur et la défend dans le cadre d'un projet de " ménagement $»^{6}$ du territoire.

11 En Argentine, en Russie, en Espagne, en Afrique de l'Ouest, la référence au marasme économique et social comme moteur et facteur de nouveaux agencements entre villes et campagnes est explicite, et très prégnante la nécessité d'assurer la subsistance alimentaire, le revenu et l'emploi. Les angles d'analyse et les faits étudiés sont néanmoins très différents. Presque tout oppose, par exemple, les maraîchers urbains d'Afrique de l'Ouest et les jardiniers de Russie, et en premier lieu le statut de l'activité et la destination des produits. Les premiers sont devenus des professionnels qui vendent et se trouvent de ce fait en concurrence, les seconds cultivent en famille, mangent leurs légumes et leurs fruits, et sont constitués en collectifs. Le développement et la professionnalisation de l'activité des premiers sont récents, le jardinage dans les datchas et autres potagers a résisté à la pression du régime soviétique, il a permis aux familles de traverser les cruelles 
années d'épreuves de sa dissolution, des faillites agricoles et industrielles qui l'ont accompagnée. Pour Louiza Boukharaeva et Marcel Marloie, ces "collectifs de jardins " étroitement complémentaires du logement urbain, replacés dans la grande nébuleuse du jardinage collectif à travers le monde, représentent une configuration idéale pour la vie en famille, dans le respect des critères de la durabilité. Les parcs-jardins de Séville et de Rosario s'inscrivent aussi dans cette mouvance de la recherche d'alternatives à l'univers « hautement concurrentiel au plan économique, social et environnemental » (Raúl Puente Asuero), qui impliquent les chercheurs aux côtés des acteurs-jardiniers. Différence notable entre les deux contextes : à Séville (et plus largement dans l'Espagne qui a suivi l'exemple pionnier de Miraflores), l'action est ancrée dans le social au sens large (le lien, l'éducation, le partage), à Rosario, on a eu le souci prioritaire de la socialisation des plus pauvres.

12 La ville est le lieu même où il importe d'agir. Les dynamiques agri-urbaines qui s'enclenchent aujourd'hui ont vocation d'exemplarité, elles expérimentent les voies d'un futur vivable. L'idée et le mot d'ordre selon lesquels il est à la fois nécessaire et possible de rendre la ville «fertile » vont de pair avec l'idée d'une présence et d'une intensité de nature (vivrière ou non, vitale de toute façon) indispensables pour réparer le fait urbain et la planète toute entière, les consolider. Cette représentation est fortement constituée dans les contextes où la vocation alimentaire n'est pas la première assignée à la pratique agricole urbaine, mais ne peut-on voir également, dans la construction de ceintures maraîchères et fruitières à Niamey, un rempart contre la menace de famine liée à la fragilité de l'agriculture pluviale, au sens matériel (une production intensive et des paysages bien réels) mais aussi social immatériel (une précarisation des individus)?

Plusieurs natures sont désirées, et elles coexistent plus ou moins pacifiquement : sur la petite ceinture ferroviaire à Paris, la biomasse intensivement fabriquée par les activistes du végétal, intrusive sur le ballast et ses dépendances, est critiquée par les écologues adeptes d'un suivi non interventionniste de la biodiversité. L'attitude la mieux partagée est toutefois celle d'une construction attentive des agroécosystèmes, d'une maîtrise forte des structures vivantes, d'un "pilotage» de la biodiversité (Blandin 2009). Il faut accompagner la nature pour qu'elle subsiste dans la ville d'aujourd'hui et dans l'urbanité généralisée qui se dessine, une préoccupation nettement spécifique du Nord, imprégnée d'une dimension philosophique et non dénuée, parfois, de connotation morale, par le jeu des bonnes pratiques « citoyennes » (Lizet \& Milliet 2012). En découle, chez les acteurs de l'agriculture urbaine, l'intérêt majeur pour le sol, fondement de la vie, qu'il s'agit d'élaborer ou de restaurer. Les adeptes de la permaculture rejoignent en cela le maraîchage urbain traditionnel (Trochet, Péru \& Roy 2003). Mais dans le même temps se dessine l'idée contradictoire, entre observation scientifique et idéologie, d'une certaine capacité de la nature à se réparer elle-même et plus précisément l'idée selon laquelle, s'il est vital de restaurer les « équilibres naturels " (conçus principalement en termes de flux, voire de forces ésotériques), les hommes ne seraient là que pour créer les conditions initiales de ces équilibres qui s'instaurent par eux-mêmes et portent en eux cette capacité (auto)régénératrice. Comme le souligne Laurence Baudelet, en ville bien plus manifestement qu'ailleurs, la terre, l'eau et l'air sont des supports de vie singulièrement ambigus, à la fois ressources et nuisances, suspects et convoités. Et c'est précisément dans cette difficulté à produire des vivres en ville, envers et contre tout, en se confrontant aux problèmes, notamment celui de la pollution, et en cherchant à réparer, aménager et ménager les mécanismes d'un vivant vivrier, que pourrait résider l'enjeu utopiste de 
l'agriculture-jardinage en ville dense. Les manières de se confronter à ces problèmes et les solutions techniques et politiques sont diverses. Laurence Baudelet montre bien que l'exigence de s'affronter à la réalité grandit: la pollution urbaine est mise en évidence aujourd'hui, sous toutes ses formes, avec ses effets sur la santé. On ne peut plus l'ignorer. Les pouvoirs publics appliquent le principe de précaution, mais des constructions éclairées sont aussi élaborées entre pouvoirs publics, bailleurs fonciers et citoyens.

Porté par les associations de jardinage partagé à Paris, l'enjeu utopiste pourrait cependant bien se trouver fragilisé par une politique de renforcement de la densité, le fameux «faire la ville sur la ville » pour enrayer l'étalement et le démantèlement des ceintures agricoles ${ }^{7}$. Voilà l'une des facettes, insuffisamment explorée aujourd'hui, de la problématique des relations entre villes et campagnes: dévitaliser un mouvement d'habitants pourtant reconnu et politiquement soutenu, et nier l'intérêt de ces poussées de ruralité paysagère et jardinière dans le cœur des villes. Comme le souligne l'analyse de Marta Soler, une mise en cohérence des politiques d'aménagement s'impose, avec un travail fin sur les échelles et l'articulation entre les enjeux, dans leur diversité.

\section{BIBLIOGRAPHIE}

Blandin P. 2009 - De la protection de la nature au pilotage de la biodiversité. Versailles, Quæ, 122 p.

Deleuze G. \& Guattari F. 1980 - Capitalisme et schizophrénie 2 : Mille Plateaux. Paris, éditions de Minuit, 648 p.

Dubbeling M. \& Van Veenhuizen R. 2014 - Formulation de politiques multi-agents et planifications d'actions en agriculture urbaine et périurbaine. POUR 224 (4) : 323-339.

Dubost F. 2011 - Potager des villes, potager des champs. In Bonnain R., Cloarec J. \& Dubost F. (Ed.) Ruralités contemporaines. Paris, L'Harmattan : 121-142.

Dubost F. \& Lizet B. 2003 - La nature dans la cité. De l'hygiénisme au développement durable. Communications $74: 5-18$.

Lizet B., Wolf A.-E. \& Célécia J. 1998 (1997) - Sauvages dans la ville. De l'inventaire naturaliste à l'écologie urbaine. Hommage à Paul Jovet (1896-1991), JATBA, $n^{\circ}$ spécial. Paris, Publications scientifiques du Muséum, 607 p.

Lizet B. \& Milliet J. 2012 - Animal certifié conforme. Comprendre nos relations avec le vivant. Paris, Dunod, 256 p.

Marié M. 1989 - Les terres et les mots. Paris, Méridiens Klincksieck, 214 p.

Mougeot L.J.A. 2006 - Cultiver de meilleures villes : agriculture urbaine et développement durable. Ottawa, Centre de Recherche pour le développement international (IDRC), 113 p. (Collection Focus).

Trochet J.-R., Péru Jean-Jacques \& Roy J.-M. 2003 - Jardinages en région parisienne, XVIIe-XXe siècle. Paris, Créaphis, 276 p. 
UN-Habitat 2003 - The challenge of slums: global report on human settlements. United Nations Human Settlements Programme. London, Earthscan Publications Ltd, 345 p. [En ligne] : http:// www.unhabitat.org.jo/pdf/GRHS.2003.pdf

\section{NOTES}

1. Ce colloque a été publié sous le titre Sauvages dans la ville. De l'inventaire naturaliste à l'écologie urbaine. Hommage à Paul Jovet (1896-1991) (1997). Les journées d'études consacrées aux «villes naturalisées » (17-18 novembre 2010 et 7 février 2012) étaient organisées en partenariat avec l'Agence d'urbanisme pour le développement de l'agglomération lyonnaise et le Grand parc Miribel-Jonage. La première opération de recherches était intitulée Villes naturalisées (2010-2012), la seconde, Villes vivrières (2012-2014). L'enseignement, dans le cadre de l'École doctorale, puis du M2 EDTS du Muséum, a été successivement dénommé «Villes, natures, paysages " (de 2007 à 2012, B. Lizet, F. Dubost et P. Frileux), puis « Villes vivrières » (depuis 2013, J. Blanc, B. Lizet et F. Dubost).

2. En Europe et notamment en France, le mouvement de l'AU exprime une contestation de la filière agricole et du statut écrasant des agriculteurs, une revendication de la légitimité et de la compétence d'un jardinage urbain vivrier, au sens d'une utilité multiple. L'un des enjeux et l'une des difficultés à venir pour les jardiniers urbains (de l'urbain), est le passage d'une production à caractère anecdotique et d'autoconsommation, à une activité marchande. Pour cette question du jardinage par opposition au maraîchage dans notre dossier, voir le texte d'Antoine Quenardel.

3. Les deux philosophes distinguaient la pensée en "racine " et la "pensée en rhizome», foisonnante et anarchique.

4. Voir The Rhizome: Journal of Permaculture Design, https://therhizome.wordpress.com (consulté le 08/08/2015)

5. Sous l'égide municipale : on n'y touche pas le sol, trop « culturel », on cultive en caissons.

6. Selon la belle formule de Michel Marié (1989). En septembre 2015, un groupe de jardins partagés, réalisé par le Conservatoire des Espaces Naturels Sensibles de l'Essonne grâce à l'opiniâtreté de la Mairie de Saulx-les-Chartreux, a été inauguré sur le territoire de l'étude.

7. À Paris, ville déjà très dense, le risque est réel et la lutte est permanente, même si les jardins partagés ont une réelle implantation sociale dans les quartiers (il en existe cent cinquante, et vingt-cinq dossiers sérieux sont à l'étude). Le risque d'être sacrifié au profit de projets d'urbanisme est une constante, il a toujours pesé sur les jardins familiaux au cours de leur longue histoire.

\section{AUTEURS}

\section{JULIEN BLANC}

Maître de conférences du Muséum national d'Histoire naturelle - Laboratoire d'Eco-

Anthropologie et Ethnobiologie

\section{BERNADETTE LIZET}

Directrice de recherche honoraire au CNRS - UMR 7206 Eco-Anthropologie et Ethnobiologie 
FRANÇOISE DUBOST

Directrice de recherche honoraire au CNRS 